\title{
Convolution Algebraic Structures Defined by Hardy-Type Operators
}

\author{
Pedro J. Miana, Juan J. Royo, and Luis Sánchez-Lajusticia \\ Departamento de Matemáticas e IUMA, Universidad de Zaragoza, 50009 Zaragoza, Spain \\ Correspondence should be addressed to Pedro J. Miana; pjmiana@unizar.es
}

Received 27 May 2013; Accepted 16 September 2013

Academic Editor: Jiecheng Chen

Copyright ( 2013 Pedro J. Miana et al. This is an open access article distributed under the Creative Commons Attribution License, which permits unrestricted use, distribution, and reproduction in any medium, provided the original work is properly cited.

\begin{abstract}
The main aim of this paper is to show that certain Banach spaces, defined via integral kernel operators, are Banach modules (with respect to some known Banach algebras and convolution products on $\mathbb{R}^{+}$). To do this, we consider some suitable kernels such that the Hardy-type operator is bounded in weighted Lebesgue spaces $L_{\omega}^{p}\left(\mathbb{R}^{+}\right)$for $p \geq 1$. We also show new inequalities in these weighted Lebesgue spaces. These results are applied to several concrete function spaces, for example, weighted Sobolev spaces and fractional Sobolev spaces defined by Weyl fractional derivation.
\end{abstract}

\section{Introduction}

Let $L^{p}\left(\mathbb{R}^{+}\right)$be the set of Lebesgue $p$-integrable (class of) functions $f$, that is, a measurable function $f \in L^{p}\left(\mathbb{R}^{+}\right)$when

$$
\|f\|_{p}=\left(\int_{0}^{\infty}|f(t)|^{p} d t\right)^{1 / p}<\infty
$$

for $1 \leq p<\infty$. The classical Hardy inequality establishes that

$$
\left(\int_{0}^{\infty}\left|\frac{1}{x} \int_{0}^{x} f(y) d y\right|^{p} d x\right)^{1 / p} \leq \frac{p}{p-1}\|f\|_{p}, \quad f \in L^{p}\left(\mathbb{R}^{+}\right)
$$

for $1<p<\infty$; that is, the so-called Hardy operator $\mathscr{H}$, defined by

$$
\mathscr{H} f(x):=\frac{1}{x} \int_{0}^{x} f(y) d y, \quad x \geq 0,
$$

is a bounded operator on $L^{p}\left(\mathbb{R}^{+}\right)$with $\|\mathscr{H}\| \leq p /(p-1)$ for $1<p<\infty$.

Let $L_{\mathrm{loc}}^{1}\left(\mathbb{R}^{+}\right)$be the set of locally integrable functions. Given $f, g \in L_{\text {loc }}^{1}\left(\mathbb{R}^{+}\right)$the (usual) convolution product $*$ is defined by

$$
(f * g)(t):=\int_{0}^{t} f(t-s) g(s) d s, \quad t \geq 0 .
$$

Given $f \in L^{1}\left(\mathbb{R}^{+}\right)$and $g \in L^{p}\left(\mathbb{R}^{+}\right)$, then $f * g \in L^{p}\left(\mathbb{R}^{+}\right)$ and

$$
\|f * g\|_{p} \leq\|f\|_{1}\|g\|_{p}
$$

for $1 \leq p \leq \infty$. Note that the Hardy operator may be written in the following way:

$$
\mathscr{H} f(x)=\frac{1}{\int_{0}^{x} \chi_{(0, \infty)}(t) d t}\left(f * \chi_{(0, \infty)}\right)(x), \quad x \geq 0,
$$

and the Hardy inequality may be written by

$$
\left\|\frac{1}{\chi_{(0, \infty)} * \chi_{(0, \infty)}} f * \chi_{(0, \infty)}\right\|_{p} \leq \frac{p}{p-1}\|f\|_{p}, \quad f \in L^{p}\left(\mathbb{R}^{+}\right),
$$

where $\chi_{(0, \infty)}$ is the characteristic function in the interval $(0, \infty)$. In fact, it is also known that if $j_{\alpha}(t):=t^{\alpha-1} /(\Gamma(\alpha))$ for $\alpha>0$, then

$$
\begin{aligned}
& \left\|\frac{1}{\chi_{(0, \infty)} * j_{\alpha}} f * j_{\alpha}\right\|_{p} \\
& \leq \frac{\Gamma(\alpha+1) \Gamma(1-1 / p)}{\Gamma(\alpha+1-1 / p)}\|f\|_{p}, \quad f \in L^{p}\left(\mathbb{R}^{+}\right),
\end{aligned}
$$


for $1<p<\infty$; see [1, Theorem 329]. From this point, there exists a wide literature about weighted inequalities of Hardytype, in particular we mention monographs [2-4] and also [5-7].

Here, we concern to functions $k$ such that the Hardy-type operator $\mathscr{H}_{k}$, given by

$$
\mathscr{H}_{k}(f)=\frac{1}{\chi_{(0, \infty)} * k} f * k,
$$

is a bounded operator in $L^{p}\left(\mathbb{R}^{+}\right)$, that is, a weighted inequality of Hardy-type

$$
\left\|\frac{1}{\chi_{(0, \infty)} * k} f * k\right\|_{p} \leq C_{k, p}\|f\|_{p}, \quad f \in L^{p}\left(\mathbb{R}^{+}\right)
$$

holds for $1<p<\infty$. This kind of inequality may be considered as a weighted inequality for Hardy-Volterra integral operators; see [3, Section 9.B] and [6, Section 4]. Under some sufficient conditions (about the integrability of $k$ ), it is possible to conclude that the operator $\mathscr{H}_{k}$ is bounded; see Theorem 5. The proof of this result is short and elegant and is inspired by the original Hardy inequality's proof (see [3, page 24]).

We use the boundedness of operator $\mathscr{H}_{k}$ (or its adjoint) to show our main result in the Section 3 Theorem 13. In Section 4, we apply our results to some concrete function spaces which, in fact, are modules for certain Banach algebras, in particular weighted Sobolev spaces, weighted fractional Sobolev spaces, and scattering Sobolev spaces. We also give some final remarks and comments about further studies.

In the Appendix, we present some new results in weighted Lebesgue spaces $L_{K}^{p}\left(\mathbb{R}^{+}\right)$for $p \geq 1$, (with $K$ satisfying some integrability conditions, as the doubling condition or the Ariño-Muckenhoupt condition; see Theorems A.2 and A.9). These results are also essential in the proof of Theorem 13.

As we have commented, our principal aim in this paper is to introduce some Banach spaces $\mathscr{T}_{k}^{p}\left(\mathbb{R}^{+}\right)$(for $1<p<\infty$ ) and to show that they are modules for the corresponding Banach algebras $\mathscr{T}_{k}^{l}\left(\mathbb{R}^{+}\right)$: given $f \in \mathscr{T}_{k}^{p}\left(\mathbb{R}^{+}\right)$and $g \in$ $\mathscr{T}_{k}^{1}\left(\mathbb{R}^{+}\right)$, then $f * g \in \mathscr{T}_{k}^{p}\left(\mathbb{R}^{+}\right)$(Theorem 13(i)). In the particular case of $k=j_{\alpha}$ for some $\alpha>0$, we obtain that the fractional Sobolev spaces $\mathscr{T}_{j_{\alpha}}^{p}\left(\mathbb{R}^{+}\right)(1<p<\infty)$ are modules for the corresponding Banach algebras $\mathscr{T}_{j_{\alpha}}^{1}\left(\mathbb{R}^{+}\right)$ (Corollary 16). Similar results hold for other convolution products, as the dual convolution product $\circ$, as follows:

$$
(f \circ g)(t):=\int_{t}^{\infty} f(s-t) g(s) d s, \quad t \geq 0, f, g \in L^{1}\left(\mathbb{R}^{+}\right),
$$

(Corollary 12) and the cosine convolution product $*_{c}$, as follows:

$$
f *{ }_{c} g:=\frac{1}{2}(f * g+f \circ g+g \circ f), \quad f, g \in L^{1}\left(\mathbb{R}^{+}\right),
$$

(Theorem 13(ii)).
Note that subalgebras $\mathscr{T}_{k}^{1}\left(\mathbb{R}^{+}\right)$(contained in $L^{1}\left(\mathbb{R}^{+}\right)$and depending of a function $k \in L_{\text {loc }}^{1}\left(\mathbb{R}^{+}\right)$) were recently introduced in [8]. Some aspects of these Banach algebras (for $k=j_{\alpha}$ with $\alpha>0$ ) were studied in [9, 10]. These algebras (for the $*$ convolution) are canonical to define some algebra homomorphisms whose kernels are $k$-convoluted semigroups; see [8, Theorem 5.5]. Roughly speaking, given $X$ a Banach space and $\mathscr{B}(X)$ the set of linear and bounded operators, a $k$-convoluted semigroup, $\left(S_{k}(t)\right)_{t>0} \subset \mathscr{B}(X)$, may be thought as a "regularization by $k$ " of a $C_{0}$-semigroup $(S(t))_{t \geq 0}$ (possibly unbounded operators) acting on $X$, as follows:

$$
S_{k}(t) x=\int_{0}^{t} k(t-s) S(s) x d s, \quad \text { for some } x \in X .
$$

For $k=j_{\alpha}$, the Banach algebra $\mathscr{T}_{j_{\alpha}}^{1}\left(\mathbb{R}^{+}\right)$was already introduced in [9] where the authors gave its connection with $\alpha$-times integrated semigroups.

Given $p \geq 1$, it is said that $p^{\prime} \geq 1$ is its conjugate exponent if $1 / p+1 / p^{\prime}=1$. For $p=1$, we follow the usual convention $p^{\prime}=\infty$. In many occasions throughout this paper, we will use the variable constant convention, in which $C$ denotes a constant which may not be the same in different lines. Subindexes in the constant will emphasize that it depends on parameters or functions.

\section{Convolution and Hardy-Type Operators}

Given $\omega: \mathbb{R}^{+} \rightarrow \mathbb{R}^{+}$as a measurable function and $1 \leq p<$ $\infty$, let $L_{\omega}^{p}\left(\mathbb{R}^{+}\right)$be the set of weighted Lebesgue $p$-integrable functions $f$, that is, $f$ is a measurable function and

$$
\|f\|_{p, \omega}:=\left(\int_{0}^{\infty}|\omega(t) f(t)|^{p} d t\right)^{1 / p}<\infty .
$$

(in fact $L_{\omega}^{p}\left(\mathbb{R}^{+}\right)$is formed by a class of functions which are equal except on Lebesgue null sets). For $p=\infty, L_{\omega}^{\infty}\left(\mathbb{R}^{+}\right)$is the set of Lebesgue measurable (class of) functions such that

$$
\|f\|_{\infty, \omega}:=\operatorname{ess} \sup _{t>0}|\omega(t) f(t)|<\infty .
$$

In the case $\omega(t)=1$ for $t>0$, we simplify this notation and write $L^{p}\left(\mathbb{R}^{+}\right)$and $\|\quad\|_{p}$ as in Section 1 .

If $\omega(t+s) \leq C \omega(t) \omega(s)$, a.e. for $t, s \geq 0$ and $C>0$, then $f * g \in L_{\omega}^{p}\left(\mathbb{R}^{+}\right)$and

$$
\|f * g\|_{p, \omega} \leq C\|f\|_{1, \omega}\|g\|_{p, \omega}
$$

for $f \in L_{\omega}^{1}\left(\mathbb{R}^{+}\right)$and $g \in L_{\omega}^{p}\left(\mathbb{R}^{+}\right)$; if $\omega(t-s) \leq C \omega(t) \omega(s)$ a.e. for $t \geq s \geq 0$ and $C>0$, then $f \circ g \in L_{\omega}^{p}\left(\mathbb{R}^{+}\right)$and

$$
\|f \circ g\|_{p, \omega} \leq C\|f\|_{1, \omega}\|g\|_{p, \omega}
$$

for $f \in L_{\omega}^{1}\left(\mathbb{R}^{+}\right)$and $g \in L_{\omega}^{p}\left(\mathbb{R}^{+}\right)$; see [11]. We prove similar inequalities in the next theorem.

We use the following notation. Let $\left(X,\|\cdot\|_{X}\right),\left(Y,\|\cdot\|_{Y}\right)$, and $\left(Z,\|\cdot\|_{Z}\right)$ be three Banach algebras and $\odot$ a binary operation $\odot: X \times Y \rightarrow Z$. We write $X \odot Y \hookrightarrow Z$ to mean that there exists $M>0$ such that $\|x \odot y\|_{Z} \leq M\|x\|_{X}\|y\|_{Y}$ for all $x \in X$ and $y \in Y$. 
Theorem 1. Let $\omega: \mathbb{R}^{+} \rightarrow \mathbb{R}^{+}$be a nonnegative and nondecreasing a.e. function and $1 \leq p<\infty$. Then

(i) $L^{p}\left(\mathbb{R}^{+}\right) \circ L_{\omega}^{1}\left(\mathbb{R}^{+}\right) \hookrightarrow L_{\omega}^{p}\left(\mathbb{R}^{+}\right)$;

(ii) $L^{1}\left(\mathbb{R}^{+}\right) \circ L_{\omega}^{p}\left(\mathbb{R}^{+}\right) \hookrightarrow L_{\omega}^{p}\left(\mathbb{R}^{+}\right)$.

Proof. (i) Let $f \in L^{p}\left(\mathbb{R}^{+}\right)$and $g \in L_{\omega}^{1}\left(\mathbb{R}^{+}\right)$; then

$$
\begin{aligned}
\| f & \circ g \|_{p, \omega} \\
& \leq\left(\int_{0}^{\infty}\left(\int_{t}^{\infty}|f(s-t)||g(s)| \omega(s) d s\right)^{p} d t\right)^{1 / p} \\
& =\left\|t \mapsto \int_{0}^{\infty} \chi_{(t, \infty)}(s)|f(s-t)| \omega(s)|g(s)| d s\right\|_{p} \\
& \leq \int_{0}^{\infty}\left\|t \mapsto \chi_{(t, \infty)}(s)|f(s-t)|\right\|_{p} \omega(s)|g(s)| d s \\
& =\int_{0}^{\infty}|g(s)| \omega(s)\left(\int_{0}^{s}|f(s-t)|^{p} d t\right)^{1 / p} d s \leq\|f\|_{p}\|g\|_{1, \omega},
\end{aligned}
$$

where we have used Minkowski's integral inequality.

(ii) Let $f \in L^{1}\left(\mathbb{R}^{+}\right)$and $g \in L_{\omega}^{p}\left(\mathbb{R}^{+}\right)$. We use similar ideas as in (i) to obtain

$$
\begin{aligned}
& \|f \circ g\|_{p, \omega} \\
& \quad \leq\left(\int_{0}^{\infty}\left(\int_{t}^{\infty} \omega(s)|f(s-t)||g(s)| d s\right)^{p} d t\right)^{1 / p} \\
& \quad=\left(\int_{0}^{\infty}\left(\int_{0}^{\infty}|f(u)||g(u+t)| \omega(u+t) d u\right)^{p} d t\right)^{1 / p} \\
& \quad \leq \int_{0}^{\infty}|f(u)|\left(\int_{u}^{\infty}|g(s)|^{p} \omega^{p}(s) d s\right)^{1 / p} d u \leq\|f\|_{1}\|g\|_{p, \omega}
\end{aligned}
$$

and we conclude the result.

As we have mentioned in Section 1, the Hardy inequality has been deeply studied, from the original one [12] to recent works, see; for example, the monograph [2] and the references therein. In [2, Theorem 2.10], authors characterize some particular functions $k$ [2, Definition 2.5] such that inequality (20) holds. See also [4, Section I.11.1]. Note that our approach does not fall into all these studies.

Definition 2. Let $p \geq 1$, let $k \in L_{\text {loc }}^{1}\left(\mathbb{R}^{+}\right)$be a nonnegative measurable function, and let $K=\chi_{(0, \infty)} * k$.

(i) We say that $k$ satisfies the $(\mathrm{HC})_{p}$ condition (Hardytype condition) if there exists $C_{k, p}>0$ such that

$$
\|k * g\|_{p, 1 / K} \leq C_{k, p}\|g\|_{p}, \quad \forall g \in L^{p}\left(\mathbb{R}^{+}\right) .
$$

(ii) We say that $k$ satisfies the $(\mathrm{dHC})_{p}$ condition (dual Hardy-type condition) if there exists $C_{k, p}^{\prime}>0$ such that

$$
\|k \circ f\|_{p} \leq C_{k, p}^{\prime}\|f\|_{p, K}, \quad \forall f \in L_{K}^{p}\left(\mathbb{R}^{+}\right) .
$$

For $p=\infty$, inequality (20) holds for any measurable and positive function $k \in L_{\mathrm{loc}}^{1}\left(\mathbb{R}^{+}\right)$. Similarly, for $p=1$, inequality (21) holds for any measurable and positive function $k \in L_{\text {loc }}^{1}\left(\mathbb{R}^{+}\right)$(without additional conditions). However, for $p=1$ and $k=\chi_{(0, \infty)}$, inequality (20) does not hold: take $g(y)=\chi_{(0,1)}(y)(1 / \sqrt{y})$.

The products $*$ and $\circ$ are dual convolution products in the following sense: the equality

$$
\int_{0}^{\infty}(k * f)(t) g(t) d t=\int_{0}^{\infty}(k \circ g)(s) f(t) d t
$$

holds for some "good" functions $k, f$, and $g$. In fact the following theorem may be present in terms of the boundedness of the Hardy-type operator $\mathscr{H}_{k}$ and its adjoint.

Theorem 3. Let $k$ be a nonnegative measurable function, and let $1<p<\infty$. Then $k$ satisfies the $(H C)_{p}$ condition if and only if $k$ satisfies the $(d H C) p_{p^{\prime}}$ condition for $p^{\prime}$ the conjugate exponent of $p$.

Proof. Suppose that $k$ satisfies the $(\mathrm{HC})_{p}$ condition. Take $f \in$ $L_{K}^{p^{\prime}}\left(\mathbb{R}^{+}\right)$and let

$$
\tilde{f}(x):=\int_{x}^{\infty} k(y-x) f(y) d y=(k \circ f)(x), \quad x \geq 0 .
$$

Let $g \in L^{p}\left(\mathbb{R}^{+}\right)$. Then

$$
\begin{aligned}
& \|\tilde{f} g\|_{1} \\
& \quad \leq \int_{0}^{\infty}\left(\int_{0}^{y} k(y-x)|g(x)| d x\right)|f(y)| d y \\
& \leq\left(\int_{0}^{\infty} \frac{1}{\left(\int_{0}^{y} k(\tau) d \tau\right)^{p}}(k *|g|(y))^{p} d y\right)^{1 / p} \\
& \quad \times\left(\int_{0}^{\infty}\left(\int_{0}^{y} k(\tau) d \tau\right)^{p^{\prime}}|f(y)|^{p^{\prime}} d y\right)^{1 / p^{\prime}} \\
& \leq C_{k, p}\|g\|_{p}\left(\int_{0}^{\infty}\left(k * \chi_{(0, \infty)}(y)|f(y)|\right)^{p^{\prime}} d y\right)^{1 / p^{\prime}} \\
& =C_{k, p}\|g\|_{p}\|f\|_{p^{\prime}, K},
\end{aligned}
$$

where Fubini's theorem has been applied in the first equality, Hölder's inequality in the second one, and the $(\mathrm{HC})_{p}$ condition in the third one. This implies that $k \circ f \in L^{p^{\prime}}\left(\mathbb{R}^{+}\right)$, $\|k \circ f\|_{p^{\prime}} \leq C_{k, p}\|f\|_{p^{\prime}, K}$, and $k$ satisfies the $(\mathrm{dHC})_{p^{\prime}}$ condition. Similarly, we prove the converse result. 
Example 4. (i) It is well-known that the function $j_{\alpha}(t):=$ $t^{\alpha-1} / \Gamma(\alpha)$ for $\alpha>0$ satisfies the $(\mathrm{HC})_{p}$; that is,

$$
\begin{aligned}
& \left\|\frac{1}{\chi_{(0, \infty)} * j_{\alpha}} f * j_{\alpha}\right\|_{p} \\
& \quad \leq \frac{\Gamma(\alpha+1) \Gamma(1-1 / p)}{\Gamma(\alpha+1-1 / p)}\|f\|_{p}, \quad f \in L^{p}\left(\mathbb{R}^{+}\right),
\end{aligned}
$$

for $1<p<\infty$; in fact; the constant $\Gamma(\alpha+1) \Gamma(1-1 / p) / \Gamma(\alpha+$ $1-1 / p)$ is optimal in this inequality, see [1, Theorem 329].

By Theorem 3, the function $j_{\alpha}$ also verifies $(\mathrm{dHC})_{p}$ for $1<$ $p<\infty$. In fact, this is a well-known result which may be found in [1, Theorem 329, page 245],

$$
\begin{aligned}
& \int_{0}^{\infty}\left(\int_{x}^{\infty} \frac{(t-x)^{\alpha-1}}{\Gamma(\alpha)}|f|(t) d t\right)^{p} d x \\
& \quad \leq\left(\frac{\Gamma(\alpha+1) \Gamma(1 / p)}{\Gamma(\alpha+1 / p)}\right)^{p} \int_{0}^{\infty}\left(\frac{x^{\alpha}}{\Gamma(\alpha+1)}|f|(x)\right)^{p} d x .
\end{aligned}
$$

Also the constant $\Gamma(\alpha+1) \Gamma(1 / p) / \Gamma(\alpha+1 / p)$ is optimal in the above inequality ([1, Theorem 329, page 245$])$.

(ii) The characteristic function $\chi_{(0,1)}$ satisfies the $(\mathrm{HC})_{p}$ for $1<p<\infty$. Note that $\chi_{(0,1)} * \chi_{(0, \infty)}(t)=\min \{1, t\}$ for $t>0$, and

$$
\begin{aligned}
\left\|\frac{1}{\chi_{(0,1)} * \chi_{(0, \infty)}} \chi_{(0,1)} * f\right\|_{p}^{p} \\
\leq \int_{0}^{1}\left(\frac{1}{t} \int_{0}^{t}|f(s)| d s\right)^{p} d t \\
\quad+\int_{1}^{\infty}\left(\int_{t-1}^{t}|f(s)| d s\right)^{p} d t \\
\leq \int_{0}^{\infty}\left(\frac{1}{t} \int_{0}^{t}|f(s)| d s\right)^{p} d t \\
\quad+\int_{1}^{\infty} \int_{t-1}^{t}|f(s)|^{p} d s d t \\
\left.\leq \quad \frac{p}{p-1}\right)^{p}\|f\|_{p}^{p} \\
\quad+\int_{0}^{1}|f(s)|^{p} s d s+\int_{1}^{\infty}|f(s)|^{p} d s \\
\leq M_{p}\|f\|_{p}^{p}
\end{aligned}
$$

for $f \in L^{p}\left(\mathbb{R}^{+}\right)$. It is clear that the characteristic function $\chi_{(1, \infty)}$ does not satisfy the $(\mathrm{HC})_{p}$ for any $1<p<\infty$.

(iii) Exponential functions $\left\{e_{\lambda}: 0 \neq \lambda \in \mathbb{R}\right\}\left(e_{\lambda}(t):=e^{\lambda t}\right.$ for $t>0)$ do not satisfy the (HC) $)_{p}$ for any $1<p<\infty$. In fact, we check that $\left\{e_{\lambda}: 0 \neq \lambda \in \mathbb{R}\right\}$ do not satisfy the $(\mathrm{dHC})_{p}$ for any $1<p<\infty$. Take $\lambda, \mu>0$, we get $e_{-\lambda} \circ e_{-\mu}=(1 /(\lambda+\mu)) e_{-\mu}$ and

$$
\left\|e_{-\lambda} \circ e_{-\mu}\right\|_{p}=\frac{1}{\lambda+\mu}\left\|e_{-\mu}\right\|_{p}=\frac{1}{\lambda+\mu}\left(\frac{1}{\mu p}\right)^{1 / p} .
$$

Note that $e_{-\lambda} * \chi_{(0, \infty)}(t)=\left(1-e^{-\lambda t}\right) / \lambda$ for $t>0$ and

$$
\begin{aligned}
\left\|e_{-\mu}\right\|_{p, e_{-\lambda} * \chi_{(0, \infty)}}^{p} & =\frac{1}{\lambda^{p}} \int_{0}^{\infty} e^{-\mu p t}\left(1-e^{-\lambda t}\right)^{p} d t \\
& \leq \int_{0}^{\infty} e^{-\mu p t} t^{p} d t=\frac{\Gamma(p+1)}{(\mu p)^{p+1}}
\end{aligned}
$$

for $\mu>0$. Then there does not exist $C>0$ such that

$$
\frac{1}{\lambda+\mu}\left(\frac{1}{\mu p}\right)^{1 / p} \leq C \frac{\Gamma(p+1)^{1 / p}}{(\mu p)^{1+1 / p}}
$$

for every $\mu>0$.

Now, take $\lambda>0$ and $\mu>\lambda$. Then $e_{\lambda} \circ e_{-\mu}=(1 /(\mu-\lambda)) e_{-\mu}$ and $e_{\lambda} * \chi_{(0, \infty)}(t)=\left(e^{\lambda t}-1\right) / \lambda$ for $t>0$. Note that

$$
\begin{aligned}
\left\|e_{-\mu}\right\|_{p, e_{\lambda} * \chi_{(0, \infty)}}^{p} & =\frac{1}{\lambda^{p}} \int_{0}^{\infty} e^{-\mu p t}\left(e^{\lambda t}-1\right)^{p} d t \\
& \leq \frac{1}{\lambda^{p}} \int_{0}^{\infty} e^{-(\mu-\lambda) p t} d t=\frac{1}{\lambda^{p}} \frac{1}{(\mu-\lambda) p},
\end{aligned}
$$

and there does not exist $C^{\prime}>0$ such that

$$
\frac{1}{\mu-\lambda}\left(\frac{1}{\mu p}\right)^{1 / p} \leq C^{\prime} \frac{1}{\lambda} \frac{1}{((\mu-\lambda) p)^{1 / p}},
$$

for any $\mu>\lambda$.

The next theorem is a particular case of [6, Theorem 4.4]: the condition $A_{k, p}(r)<\infty$ is the condition (4.7) given in [6, Theorem 4.4] for $r \in(1, p)$. We have decided to include this proof to avoid the lack of completeness of the paper.

Theorem 5. Let $k$ be a nonnegative measurable function with $\int_{0}^{\varepsilon} k(x) d x>0$ for all $\varepsilon>0$, and there exists $r \in \mathbb{R}$ such that

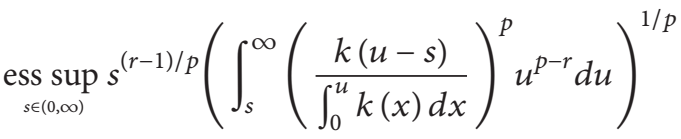

$$
\begin{aligned}
& :=A_{k, p}(r)<\infty \text {, }
\end{aligned}
$$

for some $p>1$ and $1<r<p$. Then

$$
\|g * k\|_{p, 1 / K} \leq A_{k, p}(r)\|g\|_{p}, \quad \forall g \in L^{p}\left(\mathbb{R}^{+}\right)
$$

where $K=\chi_{(0, \infty)} * k$; that is, the function $k$ satisfies the $(H C)_{p}$ condition and

$$
\left\|\mathscr{H}_{k}\right\|_{\mathscr{B}\left(L^{p}\left(\mathbb{R}^{+}\right)\right)} \leq \inf _{1<r<p}\left(\left(\frac{p-1}{p-r}\right)^{1 / p^{\prime}} A_{k, p}(r)\right),
$$

where $\mathscr{H}_{k}(g)=\left(1 / \chi_{(0, \infty)} * k\right)(k * g)$, for $g \in L^{p}\left(\mathbb{R}^{+}\right)$. 
Proof. Take $g \in L^{p}\left(\mathbb{R}^{+}\right)$, and then

$$
\begin{array}{rl}
\| g & * k \|_{p, 1 / K} \\
& =\left(\int_{0}^{\infty} \frac{1}{\left(\int_{0}^{x} k(r) d r\right)^{p}}\left(\int_{0}^{x} k(x-s)|g(s)| d s\right)^{p} d x\right)^{1 / p} \\
& =\left\|\int_{0}^{1} \frac{k(x(1-t))}{\int_{0}^{x} k(r) d r} g(x t) x d t\right\|_{p} \\
& \leq \int_{0}^{1}\left\|\int_{0}^{x} \frac{k(x(1-t))}{k(r) d r} g(x t)\right\|_{p} d t \\
& =\int_{0}^{1}\left(\int_{0}^{\infty}\left(\frac{s k((s / t)(1-t))}{t \int_{0}^{s / t} k(r) d r}\right)^{p}|g(s)|^{p} \frac{d s}{t}\right)^{1 / p} d t
\end{array}
$$

where the variable has been changed $(s=x t)$ in the first, and Minkowski's integral inequality has been used in the second step. Now, take $p^{\prime}$ the conjugate exponent of $p$ and apply the Hölder inequality and Fubini's theorem to get

$$
\begin{aligned}
\|g * k\|_{p, 1 / K} & \int_{0}^{1} t^{-(r-1) / p} t^{(r-1) / p} \\
& \times\left(\int_{0}^{\infty}\left(\frac{s k((s / t)(1-t))}{t \int_{0}^{s / t} k(r) d r}\right)|g(s)|^{p} \frac{d s}{t}\right)^{1 / p} d t \\
\leq & \left(\int_{0}^{1} t^{-p^{\prime}(r-1) / p} d t\right)^{1 / p^{\prime}} \\
& \times\left(\int_{0}^{\infty}|g(s)|^{p} s^{r-1} \int_{s}^{\infty} \frac{(k(u-s))^{p}}{\left(\int_{0}^{u} k(x) d x\right)^{p}} u^{p-r} d u d s\right)^{1 / p} \\
\leq & \left(\frac{p-1}{p-r}\right)^{1 / p^{\prime}} A_{k, p}(r)\left(\int_{0}^{\infty}|g(s)|^{p} d s\right)^{1 / p},
\end{aligned}
$$

where we have applied the assuption that $k$ satisfies (33), and we conclude that

$$
\left\|\mathscr{H}_{k}\right\|_{\mathscr{B}\left(L^{p}\left(\mathbb{R}^{+}\right)\right)} \leq \inf _{1<r<p}\left(\left(\frac{p-1}{p-r}\right)^{1 / p^{\prime}} A_{k, p}(r)\right),
$$

and the theorem is proved.
Note that inequality (33) may be written in terms of 。 product due to

$$
\begin{aligned}
k^{p} & \circ\left(\frac{h_{1-r / p}}{\left(\chi_{(0, \infty)} * k\right)}\right)^{p}(s) \\
& =\int_{s}^{\infty}\left(\frac{k(u-s)}{\int_{0}^{u} k(x) d x}\right)^{p} u^{p-r} d u, \quad s \geq 0,
\end{aligned}
$$

and $h_{1-r / p}(u)=u^{1-r / p}$ for $u>0$. In the next lemma, we give some properties of the function $A_{k, p}(r)$.

Lemma 6. Take $k$ such that $A_{k, p}(r)<\infty$ for some $p>1$ and $1<r<p$. Then

(i) $A_{k, p}\left(r^{\prime}\right) \leq A_{k, p}(r)$ for $p>r^{\prime}>r>1$;

(ii) $A_{k, q}(r) \leq\left(A_{k, p}(r)\right) /(r-1)^{(p-q) / q p}$ for $1<q<p$ and $r>1$.

Proof. (i) Take $r^{\prime}>r$, and we get

$$
\begin{aligned}
& s^{\left(r^{\prime}-1\right) / p}\left(\int_{s}^{\infty}\left(\frac{k(u-s)}{\int_{0}^{u} k(x) d x}\right)^{p} u^{p-r^{\prime}} d u\right)^{1 / p} \\
& \leq s^{(r-1) / p}\left(\int_{s}^{\infty}\left(\frac{k(u-s)}{\int_{0}^{u} k(x) d x}\right)^{p} x^{r^{\prime}-r} u^{p-r^{\prime}} d u\right)^{1 / p},
\end{aligned}
$$

for $s>0$, and then $A_{k, p}\left(r^{\prime}\right) \leq A_{k, p}(r)$. To show the part (ii), take the pair of conjugate exponents $(p / q, p /(p-q))$ with $p>$ $q$ and apply the Hölder inequality, as follows:

$$
\begin{aligned}
& \int_{s}^{\infty}\left(\frac{k(u-s)}{\int_{0}^{u} k(x) d x}\right)^{q} u^{q-r} d u \\
& \quad \leq\left(\int_{s}^{\infty}\left(\frac{k(u-s)}{\int_{0}^{u} k(x) d x}\right)^{p} u^{p-r} d u\right)^{q / p}\left(\int_{s}^{\infty} \frac{1}{u^{r}} d u\right)^{(p-q) / p},
\end{aligned}
$$

and then

$$
\begin{aligned}
& s^{(r-1) / q}\left(\int_{s}^{\infty}\left(\frac{k(u-s)}{\int_{0}^{u} k(x) d x}\right)^{q} u^{q-r} d u\right)^{1 / q} \\
& \leq \frac{s^{(r-1) / p}}{(r-1)^{(p-q) / q p}}\left(\int_{s}^{\infty}\left(\frac{k(u-s)}{\int_{0}^{u} k(x) d x}\right)^{p} u^{p-r} d u\right)^{1 / p},
\end{aligned}
$$

for $s>0$. We conclude that $A_{k, q}(r) \leq A_{k, p}(r) / r-1^{(p-q) /(q p)}$.

Example 7. (i) Let $k$ be function for which it is possible to find constants $0<m \leq M$ and $\alpha>0$ such that

$$
m \frac{t^{\alpha-1}}{\Gamma(\alpha)} \leq k(t) \leq M \frac{t^{\alpha-1}}{\Gamma(\alpha)} \text {. }
$$


We get

$$
\begin{gathered}
s^{(r-1) / p}\left(\int_{s}^{\infty} \alpha^{p} \frac{(u-s)^{(\alpha-1) p}}{u^{\alpha p}} u^{p-r} d u\right)^{1 / p} \\
=\alpha\left(\int_{0}^{\infty} \frac{x^{(\alpha-1) p}}{(1+x)^{\alpha p-p+r}} d x\right)^{1 / p} \\
=\alpha\left(\frac{\Gamma((\alpha-1) p+1) \Gamma(r-1)}{\Gamma(\alpha p-p+r)}\right)^{1 / p},
\end{gathered}
$$

and $k$ satisfies condition (33) for $r>1$ and $1 \leq p \leq 1 /(1-\alpha)$ when $0<\alpha<1$; $k$ satisfies the condition (33) for $r>1$ and $1 \leq p$ when $\alpha \geq 1$. In all these cases, we obtain

$$
\begin{aligned}
& \frac{m}{M} \alpha\left(\frac{\Gamma((\alpha-1) p+1) \Gamma(r-1)}{\Gamma(\alpha p-p+r)}\right)^{1 / p} \\
& \quad \leq A_{k, p}(r) \leq \frac{M}{m} \alpha\left(\frac{\Gamma((\alpha-1) p+1) \Gamma(r-1)}{\Gamma(\alpha p-p+r)}\right)^{1 / p},
\end{aligned}
$$

In fact condition (43) implies that the function $k$ may be written as $k=h j_{\alpha}$, where $h \in L^{\infty}\left(\mathbb{R}^{+}\right)$and $\inf _{t \geq 0} h(t) \nsupseteq 0$; then $m=\inf _{t \geq 0} h(t)$ and $M=\|h\|_{\infty}$. Particular cases are

(a) the trivial case $k:=j_{\alpha}$ for $\alpha>0$ and $A_{j_{\alpha}, p}(r)=$ $\alpha((\Gamma((\alpha-1) p+1) \Gamma(r-1)) / \Gamma(\alpha p-p+r))^{1 / p} ;$

(b) the family $k(t):=((A t+B) /(C t+D))^{\gamma} j_{\alpha}(t)$, for $A, B, C, D, \alpha, \gamma>0$. In this case,

(1) if $A D-B C<0$, then $m=(A / C)^{\gamma}$ and $M=$ $(B / D)^{\gamma}$

(2) if $A D-B C=0$, then $k=(B / D)^{\gamma} j_{\alpha}$;

(3) if $A D-B C>0$, then $m=(B / D)^{\gamma}$ and $M=$ $(A / C)^{\gamma}$.

(ii) Let $\lambda>0$, and consider functions $e_{\lambda}, e_{-\lambda}$. Then $A_{e_{-\lambda}, p}(p)=A_{e_{\lambda}, p}(p)=\infty$ for $p \geq 1$ : take $s>0$, and consider

$$
\begin{aligned}
& s^{1-1 / p}\left(\int_{s}^{\infty} \frac{e^{\lambda(u-s) p}}{\left(\int_{0}^{u} e^{\lambda r} d r\right)^{p}} d u\right)^{1 / p} \\
& =\lambda s^{1-1 / p} e^{-\lambda s}\left(\int_{s}^{\infty} \frac{1}{\left(1-e^{-\lambda u}\right)^{p}} d u\right)^{1 / p}=\infty,
\end{aligned}
$$

and we have proved the claim for the function $e_{\lambda}$. For the function $e_{-\lambda}$, note that

$$
\begin{aligned}
& \lim _{s \rightarrow \infty} s^{1-1 / p}\left(\int_{s}^{\infty} \frac{e^{-\lambda(u-s) p}}{\left(\int_{0}^{u} e^{-\lambda r} d r\right)^{p}} d u\right)^{1 / p} \\
& =\lim _{s \rightarrow \infty} s^{1-1 / p} \lambda\left(\int_{0}^{\infty} \frac{e^{-\lambda x p} d x}{\left(1-e^{-\lambda(x+s)}\right)^{p}}\right)^{1 / p}=\infty .
\end{aligned}
$$

(iii) The characteristic function $\chi_{(0,1)}$ satisfies the assumption that $A_{\chi_{(0,1)}, p}(p)=\infty$ for $p \geq 1$, such that

$$
\begin{gathered}
\sup _{s \geq 1}\left(s^{1-1 / p}\left(\int_{s}^{\infty} \frac{\chi_{(0,1)}(u-s)}{\left(\int_{0}^{u} \chi_{(0,1)}(r) d r\right)^{p}} d u\right)^{1 / p}\right) \\
=\sup _{s \geq 1}\left(s^{1-1 / p}\left(\int_{s}^{s+1} d u\right)^{1 / p}\right)=\infty .
\end{gathered}
$$

Note that the characteristic function $\chi_{(1, \infty)}$ verifies $\int_{0}^{\varepsilon} \chi_{(1, \infty)}(s) d s=0$ for $0<\varepsilon \leq 1$.

The next theorem gives the boundedness of the operator of $f \mapsto k \circ f$ in $L^{p}$-spaces. Similar results may be found in the literature, for example, [6, Theorem 4.3].

Theorem 8. Let $k$ be a nonnegative measurable function with $\int_{0}^{\varepsilon} k(x) d x>0$ for all $\varepsilon>0$, and there exists $r \in \mathbb{R}$ such that

$$
\underset{s \in(0, \infty)}{\operatorname{ess} \sup } \frac{s^{(r-1) / p}}{\int_{0}^{s} k(x) d x}\left(\int_{0}^{s} k^{p}(s-u) u^{p-r} d u\right)^{1 / p}:=B_{k, p}(r)<\infty
$$

for some $p>1$ and $p+1>r>p$. Then

$$
\|k \circ f\|_{p} \leq B_{k, p}(r)\|f\|_{p, K} \quad \forall f \in L_{K}^{p}\left(\mathbb{R}^{+}\right)
$$

where $K=\chi_{(0, \infty)} * k$; that is, the function $k$ satisfies the $(d H C)_{p}$ condition and

$$
\left\|T_{k}^{\prime}\right\|_{\mathscr{B}\left(L_{K}^{p}\left(\mathbb{R}^{+}\right), L^{p}\left(\mathbb{R}^{+}\right)\right)} \leq \inf _{p+1>r>p}\left(\left(\frac{p-1}{r-p}\right)^{1 / p^{\prime}} B_{k, p}(r)\right),
$$

where $T_{k}^{\prime}(f)=k \circ f$ for $f \in L_{K}^{p}\left(\mathbb{R}^{+}\right)$.

Proof. Take $f \in L_{K}^{p}\left(\mathbb{R}^{+}\right)$and then

$$
\begin{aligned}
\|k \circ f\|_{p} & \leq\left(\int_{0}^{\infty}\left(\int_{x}^{\infty} k(s-x)|f(s)| d s\right)^{p} d x\right)^{1 / p} \\
& =\left\|\int_{1}^{\infty} k(x(t-1))|f(x t)| x d t\right\|_{p} \\
& \leq \int_{1}^{\infty}\|k(x(t-1))|f(x t)| x\|_{p} d t \\
& =\int_{1}^{\infty}\left(\int_{0}^{\infty}(k(x(t-1))|f(x t)| x)^{p} d x\right)^{1 / p} d t
\end{aligned}
$$

where we change the variable $s=x t$ and we apply Minkowski's integral inequality. Take $p^{\prime}$ as the conjugate 
TABLE 1

\begin{tabular}{lccccc}
\hline Func. $\mid$ cond. & $(\mathrm{HC})_{p}$ & $(d \mathrm{HC})_{p}$ & $(\mathrm{DC})$ & $(\mathrm{DIC})$ & $(\mathrm{AMC})_{p}$ \\
\hline$j_{\alpha}$ & $p>1$ & $p \geq 1$ & $\checkmark$ & $\checkmark$ & $p>1$ \\
$\chi_{(0,1)}$ & $p>1$ & $p \geq 1$ & $\checkmark$ & $\checkmark$ & $p>1$ \\
$e_{-\lambda}$ & $\times$ & $\times$ & $\checkmark$ & $\checkmark$ & $p>1$ \\
$e_{\lambda}$ & $\times$ & $\times$ & $\times$ & $\checkmark$ & $\times$ \\
$\chi_{(1, \infty)}$ & $\times$ & $\times$ & $\times$ & $\times$ & $\times$ \\
\hline
\end{tabular}

for $\alpha, \lambda>0$.

exponent of $p$ and apply the Hölder inequality and Fubini's theorem to get

$$
\begin{aligned}
\|k \circ f\|_{p} & \\
\leq & \int_{1}^{\infty} t^{-(r-1) / p} t^{(r-1) / p} \\
& \times\left(\int_{0}^{\infty}(k(x(t-1))|f(x t)| x)^{p} d x\right)^{1 / p} d t \\
\leq & \left(\int_{1}^{\infty} t^{-(r-1) p^{\prime} / p} d t\right)^{1 / p^{\prime}} \\
& \times\left(\int_{0}^{\infty}|f(s)|^{p} s^{r-1} \int_{0}^{s}(k(s-u))^{p} u^{p-r} d u d s\right)^{1 / p} \\
\leq & \left(\frac{p-1}{r-p}\right)^{1 / p^{\prime}} B_{k, p}(r)\left(\int_{0}^{\infty}|f(s)|^{p}\left(\int_{0}^{s} k(x) d x\right)^{p} d s\right)^{1 / p},
\end{aligned}
$$

where we have changed the variable and applied the assumption that $k$ satisfies (49). We conclude that

$$
\left\|T_{k}^{\prime}\right\|_{\mathscr{B}\left(L^{p}\left(\mathbb{R}^{+}\right)\right)} \leq \inf _{p+1>r>p}\left(\left(\frac{p-1}{r-p}\right)^{1 / p^{\prime}} B_{k, p}(r)\right),
$$

and the theorem is shown.

To finish this section, we present Table 1 where you may find functions and their behavior with respect to several conditions considered in this section (condition $(\mathrm{HC})_{p}$ and $\left.(\mathrm{dHC})_{p}\right)$ and in the Appendix (conditions (DC), (DIC), and $\left.(\mathrm{AMC})_{p}\right)$.

\section{Convolution Banach Modules $\mathscr{T}_{k}^{p}\left(\mathbb{R}^{+}\right)$}

In the beginning of this section, we collect some definitions and properties that will be used throughout this section. We will denote by $\mathscr{D}_{+}$the set of $\mathscr{C}^{(\infty)}$ functions with compact support on $[0, \infty)$. We write by $\operatorname{supp}(k)$ as the usual support of the function $k$ and the condition $0 \in \operatorname{supp}(k)$ is equivalent to suppose that the function $k$ is not identically zero on $[0, \varepsilon)$ for all $\varepsilon>0$.

Let $k \in L_{\text {loc }}^{1}\left(\mathbb{R}^{+}\right)$be such that $0 \in \operatorname{supp}(k)$. We define the operator $T_{k}^{\prime}: \mathscr{D}_{+} \rightarrow \mathscr{D}_{+}$given by $f \mapsto T_{k}^{\prime}(f):=k \circ f$. (i) Then $T_{k}^{\prime}: \mathscr{D}_{+} \rightarrow \mathscr{D}_{+}$is an injective, linear, and continuous homomorphism such that

$$
T_{k}^{\prime}(f \circ g)=f \circ T_{k}^{\prime}(g), \quad f, g \in \mathscr{D}_{+} .
$$

(ii) The map $T_{k}^{\prime}$ extends to a linear and continuous map from $L_{K}^{1}\left(\mathbb{R}^{+}\right)$to $L^{1}\left(\mathbb{R}^{+}\right)$, which we denote again by $T_{k}^{\prime}: L_{K}^{1}\left(\mathbb{R}^{+}\right) \rightarrow L^{1}\left(\mathbb{R}^{+}\right)$such that $\left\|T_{k}^{\prime}\right\| \leq 1$.

See [8, Theorem 2.5]. Then we define the space $\mathscr{D}_{k}$ by $\mathscr{D}_{k}:=T_{k}^{\prime}\left(\mathscr{D}_{+}\right)$and the map $W_{k}: \mathscr{D}_{k} \rightarrow \mathscr{D}_{+}$by

$$
\begin{aligned}
f(t) & =T_{k}^{\prime}\left(W_{k}(f)\right)(t) \\
& =\int_{t}^{\infty} k(s-t) W_{k} f(s) d s, \quad f \in \mathscr{D}_{k}, t \geq 0 ;
\end{aligned}
$$

see [8] for more details.

Example 9. (i) Take $\alpha>0$ and $k=j_{\alpha}$; the map $W_{j_{\alpha}}$ is the Weyl fractional derivative of order $\alpha, W_{\alpha}$, and $\mathscr{D}_{j_{\alpha}}=\mathscr{D}_{+}$; note that for $\alpha \in \mathbb{N}, W_{\alpha}=(-1)^{\alpha} d^{\alpha} / d t^{\alpha}$, the $\alpha$-iterate of usual derivation; see more details, for example, in $[9,13]$.

(ii) Given $\alpha>0$ and $z \in \mathbb{C}$, take $k=e_{z} j_{\alpha}$; we have $\mathscr{D}_{k}=$ $\mathscr{D}_{+}$and

$$
W_{j_{\alpha} e_{z}} f=e_{z} W_{\alpha}\left(e_{-z} f\right), \quad f \in \mathscr{D}_{+} .
$$

See other examples in $[8$, Section 2].

(iii) For $k=\chi_{(0,1)}$, it is straightforward to check that $T_{\chi_{(0,1)}}^{\prime}(f)(t)=\int_{t}^{t+1} f(s) d s$ for $f \in \mathscr{D}_{+}, \mathscr{D}_{\chi_{(0,1)}}=\mathscr{D}_{+}$and

$$
W_{\chi_{(0,1)}} f(t)=-\sum_{n=0}^{\infty} f^{\prime}(t+n), \quad f \in \mathscr{D}_{+}, t \geq 0 .
$$

Take $f, g \in \mathscr{D}_{k}$. Then $f * g, f \circ g, f *{ }_{c} g \in \mathscr{D}_{k}$ and

$$
\begin{aligned}
& W_{k}(f * g)(s) \\
& =\int_{0}^{s} W_{k} g(r) \int_{s-r}^{s} k(t+r-s) W_{k} f(t) d t d r \\
& \quad-\int_{s}^{\infty} W_{k} g(r) \int_{s}^{\infty} k(t+r-s) W_{k} f(t) d t d r ;
\end{aligned}
$$

$W_{k}(f \circ g)=f \circ W_{k} g ; W_{k}\left(f *{ }_{c} g\right)=(1 / 2)\left(W_{k}(f * g)+f \circ\right.$ $\left.W_{k} g+g \circ W_{k} f\right)$; see [8, Theorem 2.10].

Under some conditions of $k$, some Banach algebras under the convolution product may be considered as shown in 10 .

Theorem 10 (see [8, Theorems 3.4 and 3.5]). Let $k \in L_{\text {loc }}^{1}\left(\mathbb{R}^{+}\right)$ with $0 \in \operatorname{supp}(k)$ satisfies the doubling condition $(D C)$ and $K=\chi_{(0, \infty)} * k$. Then the integral

$$
\|f\|_{1, K}:=\int_{0}^{\infty}\left|W_{k} f(t)\right| K(t) d t, \quad f \in \mathscr{D}_{k}
$$

defines an algebra norm on $\mathscr{D}_{k}$ for the convolution product * and also for $*_{c}$. We denote by $\mathscr{T}_{k}\left(\mathbb{R}^{+}\right)$the Banach space obtained as the completion of $\mathscr{D}_{k}$ in the norm $\|\cdot\|_{1, K}$, and then we have $\mathscr{T}_{k}\left(\mathbb{R}^{+}\right) \hookrightarrow L^{1}\left(\mathbb{R}^{+}\right)$. 
These Banach algebras $\mathscr{T}_{k}(\tau)$ are the algebras for which we want to establish the module versus algebra relation. If they are somehow the analogues of $L^{1}\left(\mathbb{R}^{+}\right)$, we are going to define the Banach spaces that will act as the analogues of $L^{p}\left(\mathbb{R}^{+}\right)$, but we need some tools to do this construction.

From now on, we consider $k \in L_{\text {loc }}^{1}\left(\mathbb{R}^{+}\right)$as a nonnegative function such that $0 \in \operatorname{supp}(k)$ and $K=k * \chi_{(0, \infty)}$. Let $1<$ $p<\infty$ and suppose that $k$ verifies the $(\mathrm{dHC})_{p}$ condition. Take $F \in L_{K}^{p}\left(\mathbb{R}^{+}\right)$. The function $T_{k}^{\prime} F$, given by

$$
\begin{aligned}
T_{k}^{\prime} F(t) & =(k \circ F)(t) \\
& =\int_{t}^{\infty} k(s-t) F(s) d s, \quad \text { a.e. } t \geq 0,
\end{aligned}
$$

belongs to $L^{p}\left(\mathbb{R}^{+}\right)$; moreover, $T_{k}^{\prime}$ is a bounded operator, $T_{k}^{\prime}$ : $L_{K}^{p}\left(\mathbb{R}^{+}\right) \rightarrow L^{p}\left(\mathbb{R}^{+}\right)$, which extends the operator $T_{k}^{\prime}: \mathscr{D}_{+} \rightarrow$ $\mathscr{D}_{+}$.

Definition 11. Let $\mathscr{T}_{k}^{p}\left(\mathbb{R}^{+}\right)$denote the Banach space formed as the set $T_{k}^{\prime}\left(L_{K}^{p}\left(\mathbb{R}^{+}\right)\right)$endowed with the norm $|\|\cdot\||_{p, k}$ and obtained as the image of the norm $\|\cdot\|_{p, K}$ of $L_{K}^{p}\left(\mathbb{R}^{+}\right)$through the operator $T_{k}^{\prime}: L_{K}^{p}\left(\mathbb{R}^{+}\right) \rightarrow L^{p}\left(\mathbb{R}^{+}\right)$. For $p=1$, we keep the notation $\mathscr{T}_{k}\left(\mathbb{R}^{+}\right)$.

In accordance with Definition 11, $T_{k}^{\prime}: L_{K}^{p}\left(\mathbb{R}^{+}\right) \rightarrow$ $\mathscr{T}_{k}^{p}\left(\mathbb{R}^{+}\right)$is a surjective isometry and $\mathscr{T}_{k}^{p}\left(\mathbb{R}^{+}\right)$is a Banach space. Let $W_{k}: \mathscr{T}_{k}^{p}\left(\mathbb{R}^{+}\right) \rightarrow L_{K}^{p}\left(\mathbb{R}^{+}\right)$be the inverse isometry of $T_{k}^{\prime}$ and $W_{k}: \mathscr{T}_{k}^{p}\left(\mathbb{R}^{+}\right) \rightarrow L_{K}^{p}\left(\mathbb{R}^{+}\right)$extends the operator $W_{k}: \mathscr{D}_{k} \rightarrow \mathscr{D}_{+}$defined in the beginning of this section. Note that given a function $f \in \mathscr{T}_{k}^{p}\left(\mathbb{R}^{+}\right)$, then $f \in L^{p}\left(\mathbb{R}^{+}\right)$ and there exists a unique element in $L_{K}^{p}\left(\mathbb{R}^{+}\right)$(we denote by $W_{k} f$ ) such that

$$
\begin{aligned}
f(x) & =T_{k}^{\prime}\left(W_{k} f\right)(x) \\
& =\int_{x}^{\infty} k(y-x) W_{k} f(y) d y, \quad \text { a.e. } x \geq 0 .
\end{aligned}
$$

Then for every $f \in \mathscr{T}_{k}^{p}\left(\mathbb{R}^{+}\right)$, the norm is given by

$$
\|\| f \|_{p, k}=\left(\int_{0}^{\infty}\left|W_{k} f(t)\right|^{p} K^{p}(t) d t\right)^{1 / p} .
$$

With these ideas, it is easy to show that the continuous inclusion $\mathscr{T}_{k}^{p}\left(\mathbb{R}^{+}\right) \hookrightarrow L^{p}\left(\mathbb{R}^{+}\right)$holds.

Examples. (i) For $k=j_{\alpha}$, we write $\mathscr{T}_{(\alpha)}^{p}\left(\mathbb{R}^{+}\right)$instead of $\mathscr{T}_{j_{\alpha}}^{p}\left(\mathbb{R}^{+}\right)$, for $1 \leq p<\infty$ and

$$
\|\| f \|_{p, j_{\alpha}}:=\frac{1}{\Gamma(\alpha+1)}\left(\int_{0}^{\infty}\left|W_{\alpha} f(t)\right|^{p} t^{\alpha p} d t\right)^{1 / p} .
$$

These families of spaces may be considered as Sobolev spaces of fractional order. There is huge literature about this topic; we only mention the monographs $[13,14]$ and reference therein. However, the result about the module algebra of
$\mathscr{T}_{(\alpha)}^{p}\left(\mathbb{R}^{+}\right)$for $p \geq 1$ seems to be new; see Corollary 16. The case where $\alpha \in \mathbb{N}$ (weighted Sobolev spaces) and $p=1$ was introduced and studied in [15]; in this case,

$$
\|f\|_{1, j_{n}}:=\frac{1}{n !} \int_{0}^{\infty}\left|f^{(n)}(t)\right| t^{n} d t .
$$

See Corollary 15.

(ii) In the case $k=e_{-\lambda} j_{\alpha}$, with $\alpha, \lambda>0$ and $p \geq 1$, we obtain the Banach space $\mathscr{T}_{e_{-\lambda} j_{\alpha}}^{P}\left(\mathbb{R}^{+}\right)$embedded with the norm $\mid\|f\|_{p, e_{-\lambda} j_{\alpha}}$

$$
:=\frac{1}{\Gamma(\alpha)}\left(\int_{0}^{\infty}\left|W_{\alpha}\left(e_{\lambda} f\right)(t)\right|^{p}\left(\int_{0}^{t} s^{\alpha-1} e^{-\lambda(s+t)} d s\right)^{p} d t\right)^{1 / p} .
$$

(iii) Take $k=\chi_{(0,1)}$ and $K(t)=\int_{0}^{t} \chi_{(0,1)}(s) d s=t \chi_{(0,1)}(t)+$ $\chi_{[1, \infty)}(t)$, for $t \geq 0$. We obtain the Banach space $\mathscr{T}_{\chi_{(0,1)}^{p}}^{p}\left(\mathbb{R}^{+}\right)$ for $1 \leq p<\infty$ embedded with the norm

$$
\begin{aligned}
& \|\| f \|_{p, \chi_{(0,1)}} \\
& \quad:=\left(\int_{0}^{1}\left|\sum_{n=0}^{\infty} f^{\prime}(t+n)\right|^{p} t^{p} d t+\int_{1}^{\infty}\left|\sum_{n=0}^{\infty} f^{\prime}(t+n)\right|^{p} d t\right)^{1 / p},
\end{aligned}
$$

for $f \in \mathscr{D}_{+}$.

An easy consequence of Theorem 1 and from the embedding of $\mathscr{T}_{k}^{p}\left(\mathbb{R}^{+}\right) \hookrightarrow L^{p}\left(\mathbb{R}^{+}\right)$for $p \geq 1$, we get the next corollary.

Corollary 12. Let $k \in L_{\text {loc }}^{1}\left(\mathbb{R}^{+}\right)$be a nonnegative function such that $0 \in \operatorname{supp}(k)$ and satisfy the Hardy-type condition $(d H C)_{p}$ for some $1 \leq p<\infty$. Then

(i) $L^{p}\left(\mathbb{R}^{+}\right) \circ \mathscr{T}_{k}\left(\mathbb{R}^{+}\right) \hookrightarrow \mathscr{T}_{k}^{p}\left(\mathbb{R}^{+}\right)$, and $\mathscr{T}_{k}^{p}\left(\mathbb{R}^{+}\right) \circ$ $\mathscr{T}_{k}\left(\mathbb{R}^{+}\right) \hookrightarrow \mathscr{T}_{k}^{p}\left(\mathbb{R}^{+}\right)$

(ii) $L^{1}\left(\mathbb{R}^{+}\right) \circ \mathscr{T}_{k}^{p}\left(\mathbb{R}^{+}\right) \hookrightarrow \mathscr{T}_{k}^{p}\left(\mathbb{R}^{+}\right)$, and $\mathscr{T}_{k}\left(\mathbb{R}^{+}\right) \circ$ $\mathscr{T}_{k}^{p}\left(\mathbb{R}^{+}\right) \hookrightarrow \mathscr{T}_{k}^{p}\left(\mathbb{R}^{+}\right)$.

Now we set the main result of this section.

Theorem 13. Let $1<p<\infty$, $k$ satisfy $(H C)_{p^{\prime}},(D C)$, and $(A M C)_{p^{\prime}}$, for $p^{\prime}$ such that $1 / p+1 / p^{\prime}=1$. Then
(i) $\mathscr{T}_{k}\left(\mathbb{R}^{+}\right) * \mathscr{T}_{k}^{p}\left(\mathbb{R}^{+}\right) \hookrightarrow \mathscr{T}_{k}^{p}\left(\mathbb{R}^{+}\right)$;
(ii) $\mathscr{T}_{k}\left(\mathbb{R}^{+}\right) *_{c} \mathscr{T}_{k}^{p}\left(\mathbb{R}^{+}\right) \hookrightarrow \mathscr{T}_{k}^{p}\left(\mathbb{R}^{+}\right)$.

Proof. (i) Let $f, g \in \mathscr{D}_{k}$. According to (59),

$$
\begin{aligned}
& \left|W_{k}(f * g)(s)\right|^{p} \\
& \quad \leq C\left(\int_{0}^{s}\left|W_{k} g(r)\right| \int_{s-r}^{s} k(t+r-s)\left|W_{k} f(t)\right| d t d r\right)^{p} \\
& \quad+C\left(\int_{s}^{\infty}\left|W_{k} g(r)\right| \int_{s}^{\infty} k(t+r-s)\left|W_{k} f(t)\right| d t d r\right)^{p} .
\end{aligned}
$$


Therefore,

$$
\begin{aligned}
\|\| f * g\|\|_{p, k} & =\left(\int_{0}^{\infty}\left|W_{k}(f * g)(s)\right|^{p} K^{p}(s) d s\right)^{1 / p} \\
& \leq C(I+J),
\end{aligned}
$$

where

$$
\begin{aligned}
& I:=\left(\int_{0}^{\infty} K^{p}(s)\right. \\
& \times\left(\int_{0}^{s}\left|W_{k} g(r)\right|\right. \\
& \left.\left.\times \int_{s-r}^{s} k(t+r-s)\left|W_{k} f(t)\right| d t d r\right)^{p} d s\right)^{1 / p}, \\
& J:=\left(\int_{0}^{\infty} K^{p}(s)\right. \\
& \times\left(\int_{0}^{s}\left|W_{k} g(r)\right|\right. \\
& \left.\left.\times \int_{s}^{\infty} k(t+r-s)\left|W_{k} f(t)\right| d t d r\right)^{p} d s\right)^{1 / p} .
\end{aligned}
$$

By Minkowski’s integral inequality, we get

$$
I \leq \int_{0}^{\infty}\left|W_{k} g(r)\right|\left(I_{1}+I_{2}\right) d r
$$

where

$$
\begin{aligned}
& I_{1}:=\left(\int_{r}^{2 r} K^{p}(s)\left(\int_{s-r}^{s} k(t+r-s)\left|W_{k} f(t)\right| d t\right)^{p} d s\right)^{1 / p}, \\
& I_{2}:=\left(\int_{2 r}^{\infty} K^{p}(s)\left(\int_{s-r}^{s} k(t+r-s)\left|W_{k} f(t)\right| d t\right)^{p} d s\right)^{1 / p} .
\end{aligned}
$$

We apply Theorems A.2 and A.9 to complete the proof and get

$$
|\|f * g\||_{p, k} \leq C\left|\|f\|_{p, k}\right|\|g\|_{p, k} .
$$

(ii) We use the definition of $*_{c}$, (i) and Corollary 12.

Remark 14. For $p=1, k \in L_{\text {loc }}^{1}\left(\mathbb{R}^{+}\right)$with $0 \in \operatorname{supp}(k)$ and verifying the (DC) condition, the following embeddings

$$
\begin{aligned}
\mathscr{T}_{k}\left(\mathbb{R}^{+}\right) * \mathscr{T}_{k}\left(\mathbb{R}^{+}\right) \hookrightarrow \mathscr{T}_{k}\left(\mathbb{R}^{+}\right), \\
\mathscr{T}_{k}\left(\mathbb{R}^{+}\right) *{ }_{c} \mathscr{T}_{k}\left(\mathbb{R}^{+}\right) \hookrightarrow \mathscr{T}_{k}\left(\mathbb{R}^{+}\right),
\end{aligned}
$$

hold; see Theorem 10. Note that the condition $(\mathrm{dHC})_{1}$ and Theorem A.9 hold for $p=1$.

\section{Examples, Applications, and Final Remarks}

In this section, we apply the main theorem of this paper, Theorem 13, to several particular examples of function $k$ which have appeared before. We also give some final remarks and comments.
4.1. Weighted Sobolev Spaces. Take $n \in \mathbb{N}, p>1, j_{n}(t)=$ $t^{n-1} /(n-1)$ ! for $t>0$, and the weighted Sobolev space $\mathscr{T}_{(n)}^{p}\left(\mathbb{R}^{+}\right)$is embedding with the norm

$$
\|\| f \|_{p, j_{n}}=\frac{1}{n !}\left(\int_{0}^{\infty}\left|f^{(n)}(t)\right|^{p} t^{n p} d t\right)^{1 / p} \quad f \in \mathscr{D}_{+} .
$$

Corollary 15. The Banach space $\mathscr{T}_{(n)}^{p}\left(\mathbb{R}^{+}\right)$is a module for the algebra $\mathscr{T}_{(n)}^{1}\left(\mathbb{R}^{+}\right)$(with usual convolution $*$ or the cosine convolution $*_{c}$ ) for $1<p<\infty$.

4.2. Weighted Fractional Sobolev Spaces. Let $\alpha>0, p>1$, $j_{\alpha}(t)=t^{\alpha-1} / \Gamma(\alpha)$ for $t>0$, and the weighted fractional Sobolev space $\mathscr{T}_{(\alpha)}^{p}\left(\mathbb{R}^{+}\right)$is embedding with the norm

$$
\begin{aligned}
& \|\| f \|_{p, j_{\alpha}} \\
& \quad=\frac{1}{\Gamma(\alpha+1)}\left(\int_{0}^{\infty}\left|W_{\alpha} f(t)\right|^{p} t^{\alpha p} d t\right)^{1 / p}, \quad f \in \mathscr{D}_{+},
\end{aligned}
$$

where $W_{\alpha}$ is the Weyl fractional derivation.

Corollary 16. The Banach space $\mathscr{T}_{(\alpha)}^{p}\left(\mathbb{R}^{+}\right)$is a module for the algebra $\mathscr{T}_{(\alpha)}^{1}\left(\mathbb{R}^{+}\right)$(with usual convolution $*$ or the cosine convolution $\left.*_{c}\right)$ for $1<p<\infty$.

4.3. Scattering Sobolev Spaces. Taking the function $\chi_{(0,1)}$,

$$
W_{\chi_{(0,1)}} f(t)=-\sum_{n=0}^{\infty} f^{\prime}(t+n), \quad f \in \mathscr{D}_{+}, t \geq 0,
$$

and we consider the Banach space $\mathscr{T}_{\chi_{(0,1)}}^{p}\left(\mathbb{R}^{+}\right)$for $1 \leq p<\infty$ embedded with the norm

$$
\begin{aligned}
& \||f|\|_{p, \chi_{(0,1)}} \\
& \quad:=\left(\int_{0}^{1}\left|\sum_{n=0}^{\infty} f^{\prime}(t+n)\right|^{p} t^{p} d t+\int_{1}^{\infty}\left|\sum_{n=0}^{\infty} f^{\prime}(t+n)\right|^{p} d t\right)^{1 / p},
\end{aligned}
$$

for $f \in \mathscr{D}_{+}$.

Corollary 17. The Banach space $\mathscr{T}_{\chi_{(0,1)}^{p}}^{p}\left(\mathbb{R}^{+}\right)$is a module for the algebra $\mathscr{T}_{\chi_{(0,1)}^{1}}^{1}\left(\mathbb{R}^{+}\right)$(with the usual convolution $*$ or the cosine convolution $*_{c}$ ) for $1<p<\infty$.

Final Comments. Under some conditions of a nonnegative function $k \in L_{\text {loc }}^{1}\left(\mathbb{R}^{+}\right)$, we have introduced some function spaces which are module (for the usual and cosine convolution product) with respect to some function algebras. Now we comment on other points which might be considered in further studies, and we wish to mention here the following. 
(1) For $p=2$, the Banach space $\mathscr{T}_{k}^{2}\left(\mathbb{R}^{+}\right)$could be, in fact, a Hilbert space with the inner product

$$
\begin{array}{r}
\langle f, g\rangle:=\int_{0}^{\infty} W_{k} f(t) \overline{W_{k} g(t)}\left(\int_{0}^{t} k(s) d s\right)^{2} d t \\
f, g \in \mathscr{T}_{k}^{2}\left(\mathbb{R}^{+}\right) .
\end{array}
$$

(2) For $1<p<\infty$, the dual Banach space of $\mathscr{T}_{k}^{p}\left(\mathbb{R}^{+}\right)$ may be written by $\mathfrak{T}_{k}^{p^{\prime}}\left(\mathbb{R}^{+}\right)$embedded with the norm

\|\|$f\|\|_{p^{\prime}, k}:=\left(\int_{0}^{\infty}\left|\frac{k * f(t)}{\int_{0}^{t} k(s) d s}\right|^{p^{\prime}} d t\right)^{1 / p^{\prime}}, \quad f \in \mathfrak{T}_{k}^{p^{\prime}}\left(\mathbb{R}^{+}\right)$,

where $p^{\prime}$ is the conjugate exponent of $p$.

(3) It seems to be natural that reflexivity and interpolation properties hold in Banach spaces $\mathscr{T}_{k}^{p}\left(\mathbb{R}^{+}\right)$for $1<p<\infty$.

\section{Appendix}

\section{A. Geometric Conditions and Lebesgue Norm Inequalities}

A.1. The Doubling Condition. Let $k$ be a nonnegative measurable function. We say that $k$ satisfies (DC) (the doubling condition) if there exists $D_{k}>0$ such that

$$
\text { (DC) } \int_{0}^{2 t} k(s) d s \leq D_{k} \int_{0}^{t} k(s) d s, \quad t \geq 0 .
$$

This condition is well known in real analysis and measure theory. Note that $\left(j_{\alpha}\right)_{\alpha>0}, \chi_{(0,1)}$ or $k$ nonincreasing function (in particular $\left(e_{-\lambda}\right)_{\lambda>0}$ ) satisfies the doubling condition. However, the functions $\left(e_{\lambda}\right)_{\lambda>0}$ and $\chi_{(1, \infty)}$ do not satisfy (DC).

Lemma A.1. Let $k$ be a nonnegative measurable function such that $k \in L^{1}\left(\mathbb{R}^{+}\right), \int_{0}^{\varepsilon} k(t) d t>0$, for all $\varepsilon>0$ and there exists

$$
\lim _{\varepsilon \rightarrow 0^{+}} \frac{\int_{0}^{2 \varepsilon} k(s) d s}{\int_{0}^{\varepsilon} k(s) d s}
$$

Then $k$ satisfies (DC), in particular $j_{\alpha} e_{-\lambda}$ satisfies (DC) for $\alpha>0$ and $\lambda \geq 0$.

Proof. Define $F(t):=\int_{0}^{2 t} k(s) d s / \int_{0}^{t} k(s) d s$ for $t>0$. Note that $F$ is continuous in $(0, \infty), \lim _{t \rightarrow \infty} F(t)=1$ and there exists $\lim _{t \rightarrow 0^{+}} F(t)$. We conclude that $k$ satisfies the (DC) condition.
Theorem A.2. Let $k \in L_{\text {loc }}^{1}\left(\mathbb{R}^{+}\right)$be a nonnegative function which satisfies $(D C)$ and $(d H C)_{p}$ for some $p \geq 1$ and $K=$ $\chi_{(0, \infty)} * k$. Then there exists $C>0$ such that

(i) $\left(\int_{r}^{2 r} K^{p}(s)\left(\int_{s-r}^{s} k(t+r-s)|f(t)| d t\right)^{p} d s\right)^{1 / p} \leq$ $C K(r)\|f\|_{p, K}$

(ii) $\left(\int_{2 r}^{\infty} K^{p}(s)\left(\int_{s-r}^{s} k(t+r-s)|f(t)| d t\right)^{p} d s\right)^{1 / p} \leq$ $C K(r)\|f\|_{p, K}$,

for $r \geq 0$ and $f \in L_{K}^{p}\left(\mathbb{R}^{+}\right)$.

Proof. (i) Let $I_{1}:=\left(\int_{r}^{2 r} K^{p}(s)\left(\int_{s-r}^{s} k(t+r-s)|f(t)| d t\right)^{p} d s\right)^{1 / p}$. Then we use (DC) to get

$I_{1}$

$$
\begin{aligned}
& \leq C\left(\int_{r}^{2 r} K^{p}(r)\left(\int_{s-r}^{s} k(t+r-s)|f(t)| d t\right)^{p} d s\right)^{1 / p} \\
& =C K(r)\left(\int_{0}^{r}\left(\int_{x}^{x+r} k(t-x)|f(t)| d t\right)^{p} d x\right)^{1 / p} \\
& \leq C K(r)\left(\int_{0}^{\infty}\left(\int_{x}^{\infty} k(t-x)|f(t)| d t\right)^{p} d x\right)^{1 / p} \\
& \leq C K(r)\|f\|_{p, K^{\prime}}
\end{aligned}
$$

where we have applied the condition $(\mathrm{dHC})_{p}$ in the last inequality.

(ii) Let $I_{2}:=\left(\int_{2 r}^{\infty} K^{p}(s)\left(\int_{s-r}^{s} k(t+r-s)|f(t)| d t\right)^{p} d s\right)^{1 / p}$. We use similar ideas as in (i), in particular, that $k$ satisfies $(\mathrm{dHC})_{p}$ and (DC) to obtain

$I_{2}$

$$
\begin{aligned}
& =\left(\int_{r}^{\infty}\left[\int_{0}^{x+r} k(u) d u\right]^{p}\left(\int_{x}^{x+r} k(t-x)|f(t)| d t\right)^{p} d x\right)^{1 / p} \\
& \leq C\left(\int_{r}^{\infty}\left[\int_{0}^{x} k(y) d y\right]^{p}\left(\int_{0}^{r} k(u)|f(u+x)| d u\right)^{p} d x\right)^{1 / p} \\
& \leq C \int_{0}^{r}\left(\int_{r}^{\infty}\left[\int_{0}^{x} k(y) d y\right]^{p} k^{p}(u)|f(u+x)|^{p} d x\right)^{1 / p} d u \\
& \leq C \int_{0}^{r} k(u)\left(\int_{r+u}^{\infty}\left[\int_{0}^{s} k(y) d y\right]^{p}|f(s)|^{p} d s\right)^{1 / p} d u \\
& \leq C K(r)\|f\|_{p, K},
\end{aligned}
$$

and we conclude the result.

A.2. The Decreasing Integral Condition. Let $k \in L_{\text {loc }}^{1}\left(\mathbb{R}^{+}\right)$be a nonnegative function. We say that $k$ satisfies the decreasing 
integral condition(DIC) if $\int_{0}^{\varepsilon} k(y) d y>0$ for all $\varepsilon>0$ and there exists $C_{k}>0$ such that

(DIC)

$$
\frac{\int_{0}^{u+r} k(y) d y}{\int_{0}^{u+s} k(y) d y} \leq C_{k} \frac{\int_{0}^{r} k(y) d y}{\int_{0}^{s} k(y) d y}, \quad 0 \leq s \leq r, u \geq 0 .
$$

The (DIC) condition is a technical tool which often appears in real analysis and measure theory; see, for example, level intervals and level functions in [4, Appendix].

Proposition A.3. Let $k \in L_{\mathrm{loc}}^{1}\left(\mathbb{R}^{+}\right)$be a nonnegative function which satisfies (DC). Then $k$ satisfies (DIC).

Proof. Take $r \geq s \geq 0$ and $u>0$. In the case that $r \leq u$, we have

$$
\frac{\int_{0}^{u+r} k(y) d y}{\int_{0}^{u+s} k(y) d y} \leq \frac{\int_{0}^{2 u} k(y) d y}{\int_{0}^{u} k(y) d y} \leq D_{k} \leq D_{k} \frac{\int_{0}^{r} k(y) d y}{\int_{0}^{s} k(y) d y}
$$

and in the case that $r \geq u$,

$$
\frac{\int_{0}^{u+r} k(y) d y}{\int_{0}^{u+s} k(y) d y} \leq \frac{\int_{0}^{2 r} k(y) d y}{\int_{0}^{s} k(y) d y} \leq D_{k} \frac{\int_{0}^{r} k(y) d y}{\int_{0}^{s} k(y) d y},
$$

and we conclude the proof.

Remark A.4. Note that (DC) is not equivalent to (DIC); functions $e_{\lambda}$ for $\lambda>0$ satisfy (DIC) but not (DC).

The characteristic functions $\chi_{(0,1)}$ and $j_{\alpha} e_{-\lambda}$ (with $\alpha \geq 0$ and $\lambda>0$ ) satisfy (DIC) (in fact verify (DC); see Lemma A.1). For the characteristic function $\chi_{(1, \infty)}$, (DIC) does not hold.

Lemma A.5. Let $p, q \geq 1$ and $k \in L_{\text {loc }}^{1}\left(\mathbb{R}^{+}\right)$be a positive function. If $k$ satisfies the (DIC) condition, then

$$
\begin{gathered}
\int_{0}^{r} K^{q}(s)\left(\int_{0}^{\infty}\left(\frac{k(u+r)}{K(u+s)}\right)^{p} d u\right)^{q / p} d s \\
\leq \operatorname{CrK}^{q}(r)\left(\int_{0}^{\infty}\left(\frac{k(u+r)}{K(u+r)}\right)^{p} d u\right)^{q / p},
\end{gathered}
$$

for $r \geq 0$, where $K=\chi_{(0, \infty)} * k$.

Proof. We apply the definition of (DIC) to get

$$
\begin{aligned}
& \int_{0}^{r} K^{q}(s)\left(\int_{0}^{\infty}\left(\frac{k(u+r)}{K(u+s)}\right)^{p} d u\right)^{q / p} d s \\
& \quad \leq C \int_{0}^{r} K^{q}(s)
\end{aligned}
$$

$$
\begin{gathered}
\times\left(\int_{0}^{\infty} \frac{\left(\int_{0}^{r} k(y) d y\right)^{p}}{\left(\int_{0}^{s} k(y) d y\right)^{p}}\right. \\
\left.\times\left(\frac{k(u+r)}{\int_{0}^{u+r} k(y) d y}\right)^{p} d u\right)^{p / q} d s \\
=C^{q}(r)\left(\int_{0}^{\infty}\left(\frac{k(u+r)}{\int_{0}^{u+r} k(y) d y}\right)^{p} d u\right)^{q / p}\left(\int_{0}^{r} d s\right)
\end{gathered}
$$

for $r \geq 0$.

A.3. The Ariño-Muckenhoupt Condition. Let $k \in L_{\text {loc }}^{1}\left(\mathbb{R}^{+}\right)$ be a nonnegative function and $1<p<\infty$ with $p^{\prime}$ as its conjugate exponent. We say that $k$ satisfies $(\mathrm{AMC})_{p}$ (AriñoMuckenhoupt condition) if $\int_{0}^{\varepsilon} k(y) d y>0$ for all $\varepsilon>0$ and there exists $C_{k}>0$ such that

$$
\begin{aligned}
(\mathrm{AMC})_{p} \quad r^{1 / p^{\prime}} & \left(\int_{r}^{\infty}\left(\frac{k(u)}{\int_{0}^{u} k(y) d y}\right)^{p} d u\right)^{1 / p} \\
\leq & C_{k}, r \geq 0
\end{aligned}
$$

The well-known Ariño-Muckenhoupt theorem states that the weighted Hardy inequality

$$
\begin{gathered}
\left(\int_{0}^{\infty}\left|\int_{0}^{x} f(t) d t\right|^{p} u(x) d x\right)^{1 / p} \\
\quad \leq C\left(\int_{0}^{\infty}|f(x)|^{q} v(x) d x\right)^{1 / q}
\end{gathered}
$$

holds for $1 \leq q \leq p \leq \infty$ if and only if

$$
\sup _{r>0}\left(\int_{r}^{\infty} u(x) d x\right)^{1 / p}\left(\int_{0}^{r} v(x)^{1-q^{\prime}} d x\right)^{1 / q^{\prime}}<\infty
$$

see, for example, [3, page 44]. Note that $(\mathrm{AMC})_{p}$ is, in fact, a particular case of (A.12) for $q=p, u(x)=(k(x) / K(x))^{p}$ and $v(x)=1$ for $x \geq 0$. Then (AMC) ${ }_{p}$ holds if and only if

$$
\left\|\chi_{(0, \infty)} * f\right\|_{p, k / K} \leq C\|f\|_{p}, \quad f \in L^{p}\left(\mathbb{R}^{+}\right)
$$

for $1 \leq p<\infty$ and $K=\chi_{(0, \infty)} * k$.

Remark A.6. We just need Hölder's inequality to proof that, if $k$ satisfies $(\mathrm{AMC})_{p_{1}}$ and $(\mathrm{AMC})_{p_{2}}$, with $1 \leq p_{1} \leq p_{2}<\infty$, then $k$ satisfies $(\mathrm{AMC})_{p}$ for all $p \in\left[p_{1}, p_{2}\right]$.

The characteristic function $\chi_{(0,1)}$ satisfies $(\mathrm{AMC})_{p}$ for all $p>1$; nevertheless, $\chi_{(1, \infty)}$ does not satisfy $(\mathrm{AMC})_{p}$ for any 
$p \geq 1$. The functions $j_{\alpha} e_{-\lambda}$ for $\alpha>0$ and $\lambda \geq 0$ satisfy $(\mathrm{AMC})_{p}$ for $p>1$ as follows:

$$
\begin{aligned}
& r^{1 / p^{\prime}}\left(\int_{r}^{\infty}\left(\frac{u^{\alpha-1} e^{-\lambda u}}{\int_{0}^{u} s^{\alpha-1} e^{-\lambda s} d s}\right)^{p} d u\right)^{1 / p} \\
& =r^{1 / p^{\prime}}\left(\int_{r}^{\infty} \frac{u^{p(\alpha-1)}}{\left(\int_{0}^{u} s^{\alpha-1} e^{\lambda(u-s)} d s\right)^{p}} d u\right)^{1 / p} \\
& \leq \alpha r^{1 / p^{\prime}}\left(\int_{r}^{\infty} \frac{d u}{u^{p}}\right)^{1 / p}=\frac{\alpha}{(p-1)^{1 / p}}
\end{aligned}
$$

for $r>0$. However, $e_{\lambda}$ does not satisfy (AMC) $)_{p}$ for any $p \geq 1$.

In Lemma A.7, we prove that there does not exist a nonnegative function $k \in L_{\text {loc }}^{1}\left(\mathbb{R}^{+}\right)$such that satisfies $(\mathrm{AMC})_{1}$.

Lemma A.7. Let $k \in L_{\text {loc }}^{1}\left(\mathbb{R}^{+}\right)$be a nonnegative function such that $\int_{0}^{\varepsilon} k(r) d r>0$ for all $\varepsilon>0$. Then

$$
\int_{0}^{\infty} \frac{k(u)}{\int_{0}^{u} k(r) d r} d u=\infty .
$$

Proof. Suppose that $\int_{0}^{\infty}\left(k(u) / \int_{0}^{u} k(r) d r\right) d u<\infty$. Take $1>$ $\varepsilon^{\prime}>0$ such that $\int_{0}^{\varepsilon^{\prime}} k(r) d r>0$. Then

$$
\begin{aligned}
\infty & >\int_{0}^{\infty} \frac{k(u)}{\int_{0}^{u} k(r) d r} d u \\
& \geq \int_{0}^{\varepsilon} \frac{k(u)}{\int_{0}^{u} k(r) d r} d u \\
& \geq \int_{0}^{\varepsilon} \frac{k(u)}{\int_{0}^{\varepsilon} k(r) d r} d u=1,
\end{aligned}
$$

for any $0<\varepsilon<\varepsilon^{\prime}$. By the dominated convergence theorem, we conclude that

$$
1 \leq \lim _{\varepsilon \rightarrow 0^{+}} \int_{0}^{\varepsilon} \frac{k(u)}{\int_{0}^{u} k(r) d r} d u=0,
$$

and we conclude the proof of the lemma.

Corollary A.8. Let $p>1$, and let $p^{\prime}$ be its conjugate exponent and $k \in L_{\mathrm{loc}}^{1}\left(\mathbb{R}^{+}\right)$a positive function. If $k$ satisfies (DIC) and $(A M C)_{p}$, then

$$
\begin{aligned}
& \int_{0}^{r}\left(\int_{0}^{s} k(y) d y\right)^{p^{\prime}}\left(\int_{0}^{\infty}\left(\frac{k(u+r)}{\int_{0}^{u+s} k(y) d y}\right)^{p} d u\right)^{p^{\prime} / p} d s \\
& \quad \leq C\left(\int_{0}^{r} k(y) d y\right)^{p^{\prime}},
\end{aligned}
$$

Proof. We apply Lemma A.5 and the (AMC) $)_{p}$ condition to get the result.

Theorem A.9. Let $k \in L_{\mathrm{loc}}^{1}\left(\mathbb{R}^{+}\right)$be a nonnegative function such that satisfies $(D I C)$ and $(A M C) p_{p^{\prime}}$ for some $p^{\prime}>1$ (with $p$ its conjugate exponent) and $K=\chi_{(0, \infty)} * k$. Then there exists a constant $C>0$, such that

$$
\begin{aligned}
& \left(\int_{0}^{\infty} K^{p}(s)\left(\int_{0}^{s}|g(r)| \int_{s}^{\infty} k(t+r-s)|f(t)| d t d r\right)^{p} d s\right)^{1 / p} \\
& \quad \leq C\|f\|_{p, K}\|g\|_{1, K},
\end{aligned}
$$

for $f \in L_{K}^{p}\left(\mathbb{R}^{+}\right), g \in L_{K}^{1}\left(\mathbb{R}^{+}\right)$.

Proof. Let $J:=\left(\int_{0}^{\infty} K^{p}(s)\left(\int_{0}^{s}|g(r)| \int_{s}^{\infty} k(t+r-s)|f(t)| d t d r\right)^{p}\right.$ $d s)^{1 / p}$ for $f \in L_{K}^{p}\left(\mathbb{R}^{+}\right)$and $g \in L_{K}^{1}\left(\mathbb{R}^{+}\right)$. Then

$$
\begin{gathered}
J \leq\left(\int _ { 0 } ^ { \infty } \left(\int_{s}^{\infty}|g(r)|\left(\int_{0}^{s} k(y) d y\right)\right.\right. \\
\left.\left.\times \int_{s}^{\infty} k(t+r-s)|f(t)| d t d r\right)^{p} d s\right)^{1 / p} \\
\leq \int_{0}^{\infty}|g(r)|\left(\int_{0}^{r}\left(\int_{0}^{s} k(y) d y\right)^{p}\right. \\
\left.\times\left(\int_{s}^{\infty} k(t+r-s)|f(t)| d t\right)^{p} d s\right)^{1 / p} d r .
\end{gathered}
$$

Now, we apply Hölder's inequality to obtain

$$
\begin{aligned}
& \int_{s}^{\infty} \frac{k(t+r-s)}{\int_{0}^{t} k(y) d y}|f(t)|\left(\int_{0}^{t} k(y) d y\right) d t \\
& \quad \leq\left(\int_{s}^{\infty}\left(\frac{k(t+r-s)}{\int_{0}^{t} k(u) d u}\right)^{p^{\prime}} d t\right)^{1 / p^{\prime}}\|f\|_{p, K},
\end{aligned}
$$

and then

$$
\begin{aligned}
& J \leq\|f\|_{p, K} \\
& \times \int_{0}^{\infty}|g(r)| \\
& \quad \times\left(\int_{0}^{r}\left(\int_{0}^{s} k(y) d y\right)^{p}\right. \\
& \left.\quad \times\left(\int_{0}^{\infty}\left(\frac{k(u+r)}{\int_{0}^{u+s} k(y) d y}\right)^{p^{\prime}} d u\right)^{p / p^{\prime}} d s\right)^{1 / p} d r .
\end{aligned}
$$

for $r \geq 0$. 
Now, we apply Corollary A.8 to conclude that

$$
\begin{aligned}
J & \leq C\|f\|_{p, K} \int_{0}^{\infty}|g(r)|\left(\int_{0}^{r} k(y) d y\right) d r \\
& \leq C\|f\|_{p, K}\|g\|_{1, K} .
\end{aligned}
$$

Remark A.10. For $p=1$, inequality (A.19) holds with $k$ satisfying (DC) without any additional condition (i.e., $\left.(\mathrm{AMC})_{\infty}\right)$; we just apply the Fubini theorem.

\section{Acknowledgments}

The authors wish to thank J. E. Galé and F. J. Ruiz for a patient reading, valuable suggestions, and several references which have improved this paper. Authors have been partly supported by the Ministry of Education and Science DGI-FEDER (MTM 2010-16679), the DGA Project (E-64) "Análisis Matemático y Aplicaciones" and JIUZ-2012-CIE-12, Universidad de Zaragoza, Spain.

\section{References}

[1] G. H. Hardy, J. E. Littlewood, and G. Polya, Inequalities, Cambridge University Press, Cambridge, UK, 1964.

[2] A. Kufner and L. E. Persson, Weighted Inequalities of HardyType, Word Scientific, New Jersey, NJ, USA, 2003.

[3] A. Kufner, L. Maligranda, and L. E. Persson, The Hardy Inequality: About Its History and Some Related Results, Vydavatelsky Servis, Pilsen, Czech Republic, 2007.

[4] B. Opic and A. Kufner, Hardy-Type Inequalities, Longman Scientic \& Technical, New York, NY, USA, 1990.

[5] K. F. Andersen, "Weighted generalized Hardy inequalities for nonincreasing functions," Canadian Journal of Mathematics, vol. 43, pp. 1121-1135, 1991.

[6] S. Kaijser, L. Nikolova, L. Persson, and A. Wedestig, "Hardytype inequalities via convexity," Mathematical Inequalities and Applications, vol. 8, no. 3, pp. 403-417, 2005.

[7] F. J. Martin-Reyes and E. Sawyer, "Weighted inequalities for Riemann-Liouville fractional inte- grals of order," Proceedings of the American Mathematical Society, vol. 106, no. 3, pp. 727733, 1989.

[8] V. Keyantuo, C. Lizama, and P. J. Miana, "Algebra homomorphisms defined via convoluted semigroups and cosine functions," Journal of Functional Analysis, vol. 257, no. 11, pp. 3454-3487, 2009.

[9] J. E. Galé and P. J. Miana, "One-parameter groups of regular quasimultipliers," Journal of Functional Analysis, vol. 237, no. 1, pp. 1-53, 2006.

[10] J. E. Galé, P. J. Miana, and J. J. Royo, "Estimates of the Laplace transform on convolution Sobolev algebras," Journal of Approximation Theory, vol. 164, no. 1, pp. 162-178, 2012.

[11] P. J. Miana, "Algebra homomorphisms from cosine convolution algebras," Israel Journal of Mathematics, vol. 165, pp. 253-280, 2008.

[12] G. H. Hardy, "Notes on some points in the integral calculus, LX. An inequality between integrals," Messenger of Mathematics, vol. 54, pp. 150-156, 1925.
[13] S. G. Samko, A. A. Kilbas, and O. I. Marichev, Fractional Integrals and Derivates. Theory and Applications, Gordon-Breach, New York, NY, USA, 1993.

[14] T. Runst and W. Sickel, Sobolev Spaces of Fractional Order, Nemytskij Operatores, and Non-Linear Partial Differential Equation, Walter de Gruyter, Berlin, Germany, 1996.

[15] W. Arendt and H. Kellermann, "Integrated solutions of Volterra integrodifferential equations and applications," in Volterra Integrodifferential Equations in Banach Spaces and Applications (Trento, 1987), vol. 190 of Pitman Research Notes in Mathematics Series, pp. 21-51, Longman Science, Harlow, UK, 1989. 


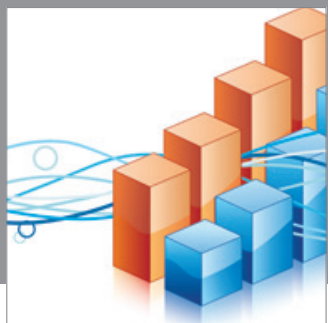

Advances in

Operations Research

mansans

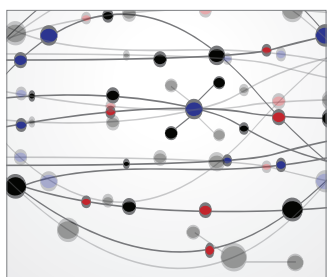

The Scientific World Journal
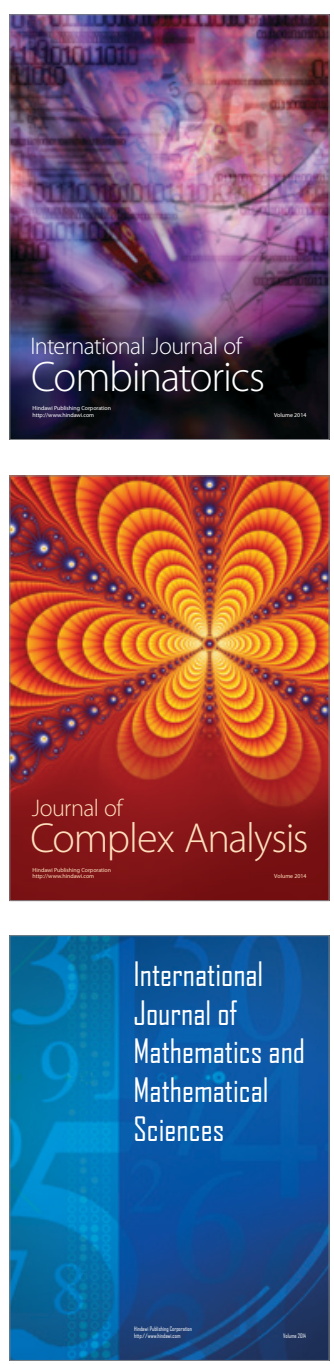
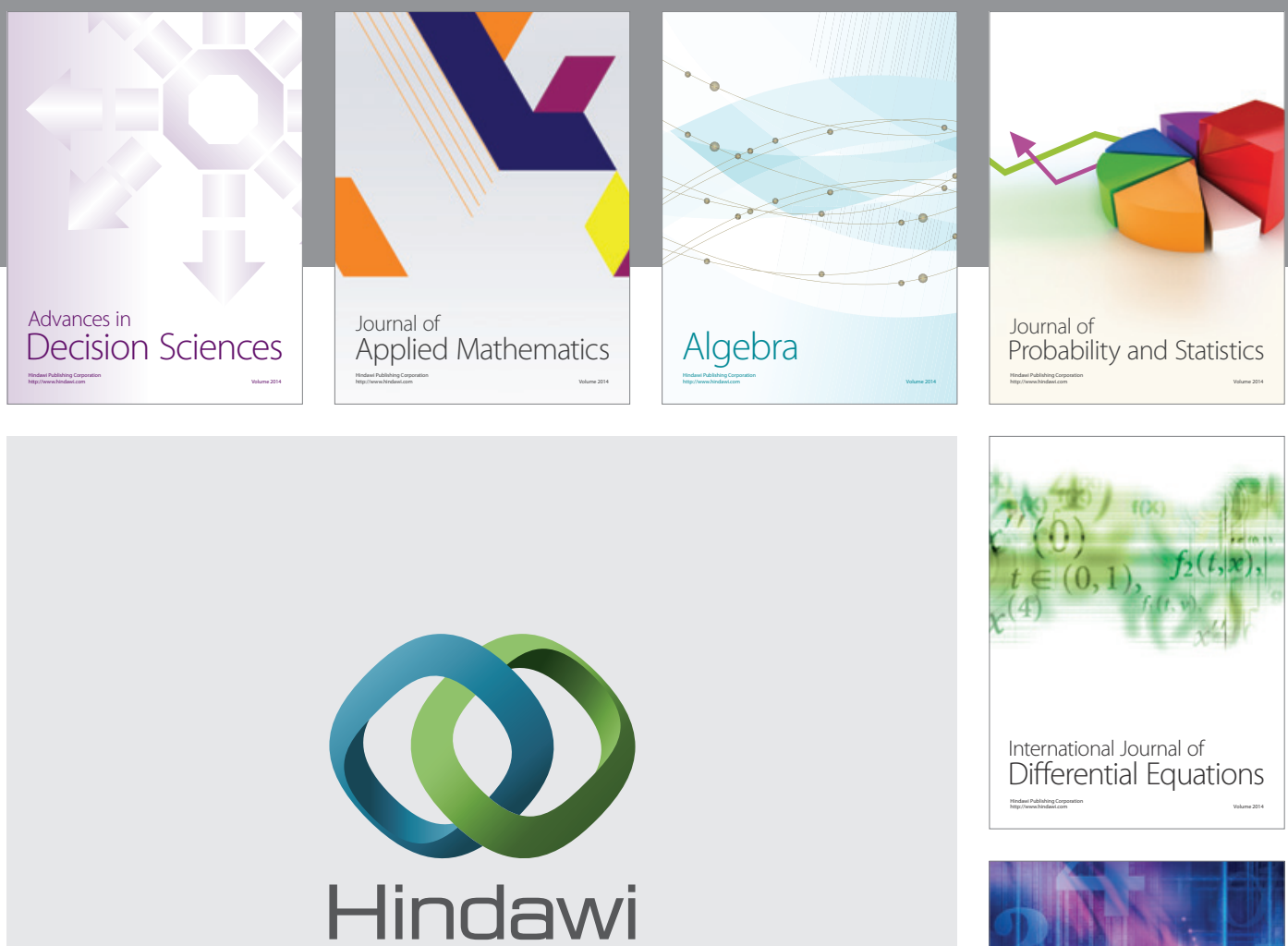

Submit your manuscripts at http://www.hindawi.com
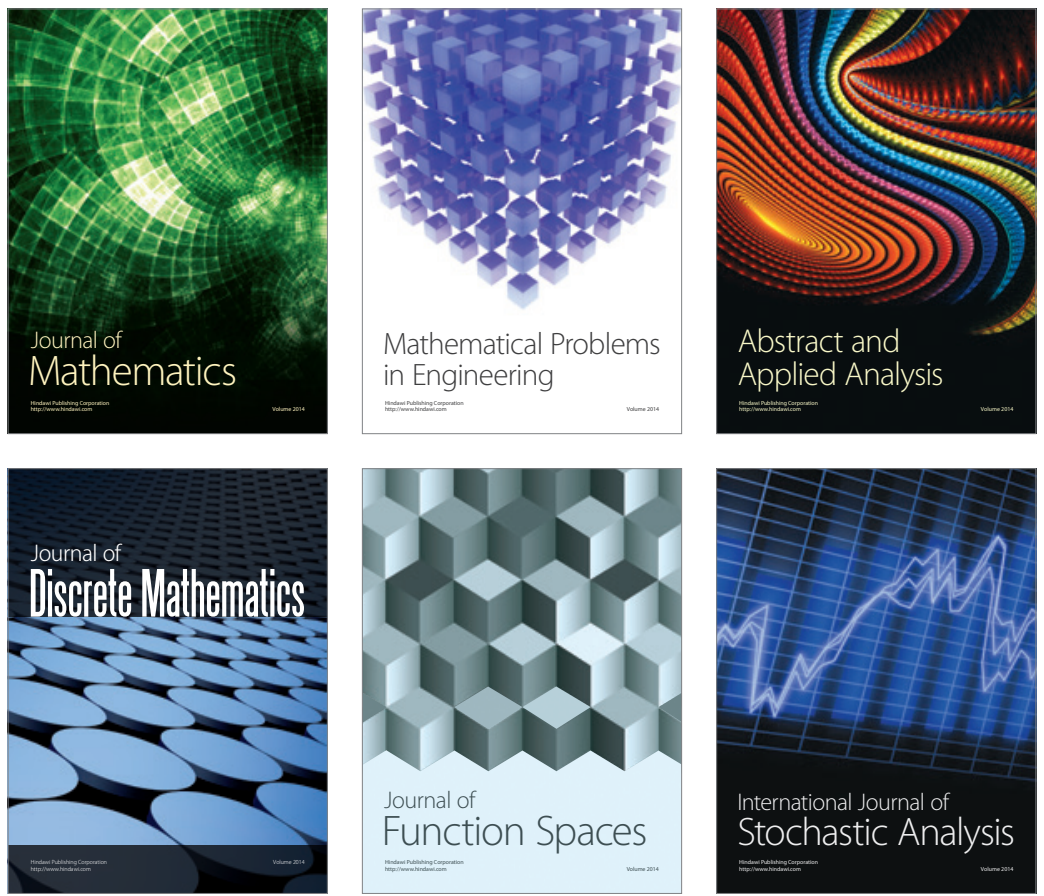

Journal of

Function Spaces

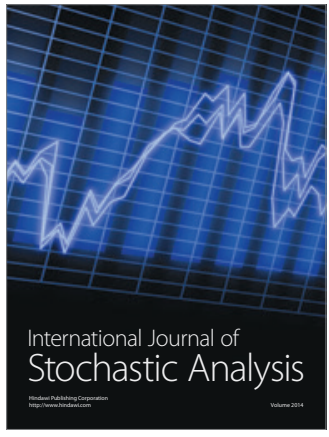

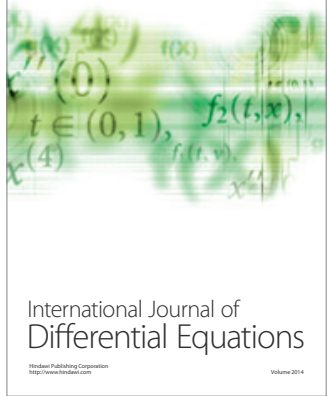
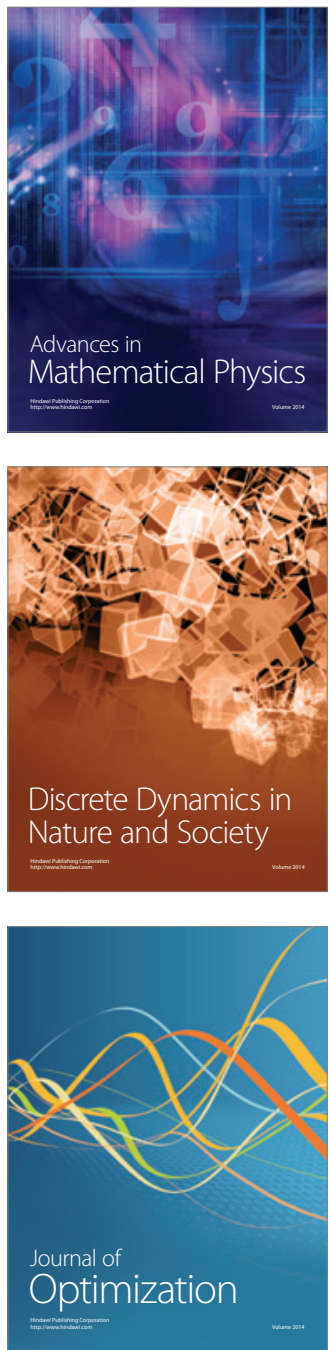